\title{
Presence of Candida zemplinina in Sicilian Musts and Selection of a Strain for Wine Mixed Fermentations
}

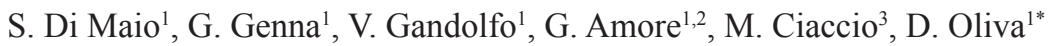 \\ (1) Istituto Regionale della Vite e del Vino (IRVV), Regione Siciliana, Via della Libertà 66, 90143, Palermo, Italy \\ (2) Stazione Zoologica “Anton Dohrn” Napoli, Villa Comunale 80121, Napoli, Italy \\ (3) Istituto di Biomedicina e Immunologia Molecolare, CNR, Via Ugo La Malfa 153, 90143, Palermo, Italy
}

Submitted for publication: September 2011

Accepted for publication: December 2011

Key words: wine fermentation, mixed fermentations, Candida zemplinina, non-Saccharomyces, sequential inoculation

\begin{abstract}
The purpose of this work was to investigate the presence of $C$. zemplinina yeasts in Sicilian musts and grapes and to identify strains of oenological interest. We report on the taxonomical reclassification of Candida yeast isolates from Sicilian musts and on the selection of one strain of oenological interest $(\mathrm{Cz3})$, based on mixed micro-fermentation experiments in sterile Nero d'Avola musts. Our results show that Candida zemplinina is abundant in Sicilian grapes and musts, and that the Cz3 strain is suitable for Candida zemplinina/Saccharomyces cerevisiae mixed fermentations. The higher glycerol content and the lower ethanol level stood out as the most promising features of the wines obtained upon sequential inoculation of the $\mathrm{Cz3}$ and (S. cerevisiae) NDA21 yeast starters. We therefore have isolated a Sicilian Cz strain endowed with very promising features for the future development of mixed fermentation protocols.
\end{abstract}

\section{INTRODUCTION}

The possibility of increasing wine complexity via mixed fermentation represents an attractive opportunity offered by recent oenological research. This exploits the contribution of some non-Saccharomyces yeast species that are present early in spontaneous fermentations. Although attractive, spontaneous fermentations are not easy to control in their progression and outcome. On the other hand, mixed fermentations, where selected non-Saccharomyces species are inoculated together with a commercial Saccharomyces strain, may be a more manageable way to increase the appeal of certain wines. Non-Saccharomyces species can synthesise compounds and enzymes that can positively affect the quality of the wines (e.g. Languet et al., 2005). Furthermore, several negative features of non-Saccharomyces yeasts are attenuated in mixed fermentations (Du Toit \& Pretorius, 2000; Anfang et al., 2009).

It has been reported that Candida zemplinina and Hanseniaspora spp. are the yeasts most frequently found in spontaneous fermentations (Andorrà et al., 2010). The higher glycerol content and the relatively low ethanol levels are the most appealing features of the wines made using Candida yeasts (Magyar \& Tóth, 2011). These have a commercial interest, since glycerol ameliorates taste, while low ethanol levels can attract a wide range of consumers. Among Candida species, C. zemplinina occurs most frequently in musts (Csoma \& Sipiczki, 2008). Compared to C. stellata, C. zemplinina yeasts have been suggested to be better suited for mixed fermentations, since they could reduce the osmotic stress imposed on Saccharomyces cells more efficiently by removing more sugar from the culture medium (Cavagna et al., 2008; Rantsiou et al., 2008).

For this paper we analysed 59 Candida zemplinina isolates from musts of autochthonous cultivars belonging to the non-Saccharomyces collection of the Istituto Regionale della Vite e del Vino (IRVV-Palermo, Italy). We present the oenological characterisation of these isolates and we report on the identification of one of these strains $(\mathrm{Cz} 3)$, which featured the best results in sequential inoculation protocols with a commercial Saccharomyces strain.

\section{MATERIALS AND METHODS}

Yeasts

The 59 Candida zemplinina isolates $(\mathrm{Cz})$ analysed in this work belong to the IRVV non-Saccharomyces collection and were previously reported as C. stellata isolates (Romancino et al., 2008). The Saccharomyces cerevisiae (Sc) strain NDA21 (Di Maio et al., 2006) belongs to the IRVV collection and is commercialised by Biospringer. The Sc yeast strains L1, L2, L3 and L4 belong to the Saccharomyces IRVV collection (Di Maio et al., 2012). The commercial Sc strains ICV-k1, EC1118, ICV-D254, RC212, QD145, Uvaferm43 and Ba11 are distributed by Lallemand; Zymaflore F10 is distributed by Laffort. The $S$. cerevisiae L404 strain belongs to the DIPROVAL collection of the University of Bologna, 
Italy. The H. uvarum $\mathrm{Hu} 03$ and M. pulcherrima Mp03 strains belong to the IRVV collection. The $C$. stellata 6714 strain was provided by DBVPG (Department of Applied Biology, Industrial Yeast Collection, University of Perugia, Italy). $C$. zemplinina and $C$. stellata yeasts were grown in malt agar and kept at $4^{\circ} \mathrm{C}$. The Saccharomyces, Hanseniaspora and Metschnikowia yeasts were cultured in Sabouraud dextrose agar (Oxoid), with $10 \mathrm{~g} / \mathrm{L}$ of yeast extract (Oxoid), at $4^{\circ} \mathrm{C}$. All cultures were renewed periodically.

\section{Molecular characterisation}

The 59 Candida isolates (IRVV) were characterised as representatives of the $C$. zemplinina species by amplifying the ITS1-5.8S-ITS2 region and by digesting it with DraI and MboI endonucleases. The C. stellata DBVPG 6714 strain was used for reference. All our isolates showed the same digestion pattern, identical to that described for C. zemplinina (Csoma \& Sipiczki, 2008). Sequencing and analysis of the D1/D2 domain of the 26S rDNA was done at the DBVPG. To distinguish the isolates, the DNA was extracted following the procedure of Querol et al. (1992); the DNA was then digested with the HaeIII and HpaII endonucleases (which allow an intra-specific distinction in the closely related $C$. stellata species) and the restriction patterns of the mt-DNA were analysed (Pramateftaki et al., 2000).

\section{Oenological characterisation}

Acetic acid production was measured according to Caridi et al. (2002). The L404 strain and the H. uvarum Hu03 strain were used for reference. $\mathrm{H}_{2} \mathrm{~S}$ production was assessed on BiGGY agar (Nickerson, 1953). L1-L4, EC1118, L404, ICV-D254, QD145, Uvaferm43, NDA21, Zymaflore F10 strains were used for reference.

ß-glucosidase activity was measured as in Strauss et al. (2001), with modifications: yeasts were seeded on arbutin with/without 200g/L glucose-fructose (1:1). No-arbutin plates were used for control. The control strains were L404 (negative), and $\mathrm{Hu} 03$ and Mp03 (positive).

Killer activity was determined as in Regodón et al. (1997), seeding the yeasts over a layer of Ba11 cells (Lallemand). The ICV-k1 and EC1118 strains were used as positive controls; the ICV-D254 and RC212 strains were used as negative controls. The killer activity of the $\mathrm{Cz}$ and NDA21 strains versus each other was evaluated by seeding each strain over a layer of the other.

Fermentative vigour was measured according to Caridi et al. (2002) in $100 \mathrm{~mL}$ of sterile must obtained by diluting concentrated must down to $20^{\circ} \mathrm{Brix}$ and adjusting the $\mathrm{pH}$ to 3.2. Fermentative vigour (weight loss due to $\mathrm{CO}_{2}$ production; average of two replicates) was measured after two and seven days at $25^{\circ} \mathrm{C}$. L404-inoculated, NDA21-inoculated and non-inoculated must were used for reference. After 12 days the musts were filtered and oenochemical analyses were performed.

$\mathrm{SO}_{2}$ tolerance (Caridi et al., 2002) and fermentative power (Zambonelli et al., 2000) were determined in the same kind of must. For $\mathrm{SO}_{2}$ tolerance, musts were supplemented with $200 \mathrm{mg} / \mathrm{L}$ potassium metabisulphite. To measure fermentative power, the musts were supplemented with glucose up to $300 \mathrm{~g} / \mathrm{L}$ sugars. Fermentative power was measured every day until the measured weight loss was less than $0.01 \mathrm{~g} / \mathrm{L}$. Measurements were performed in duplicate and average values were determined.

Fermentative activity of Czstrains in $250 \mathrm{ml}$ fermentations Nero d'Avola rosé must sample (100 mg/L potassium metabisulphite; filter sterilised) aliquots were inoculated with each one of the $\mathrm{Cz}$ strains or the NDA21 strain alone (pure fermentations $(\mathrm{P}): 2 \times 10^{6} \pm 0.2 \times 10^{6} \mathrm{cfu} / \mathrm{mL}$ ); or with each of the three $\mathrm{Cz}$ strains and the NDA21 strain (coinoculations (CI): $\mathrm{Cz}$ strains, $1.5 \times 10^{6} \pm 0.2 \times 10^{6} \mathrm{cfu} / \mathrm{mL}$; NDA21, $\left.0.5 \times 10^{6} \pm 0.2 \times 10^{6} \mathrm{cfu} / \mathrm{mL}\right)$; or with each $\mathrm{Cz}$ strains and, after five days, with NDA21 (sequential inoculations (Seq): both strains $2 \times 10^{6} \pm 0.2 \times 10^{6} \mathrm{cfu} / \mathrm{mL}$; at the time of the NDA21 strain inoculation, the $\mathrm{Cz}$ strains were at $61 \times 10^{6}$ $\pm 3.5 \times 10^{6} \mathrm{cfu} / \mathrm{mL}$ ). The yeasts came from monocultures in the same kind of must. Fermentations were conducted at $28^{\circ} \mathrm{C}$; every day, microbiological analyses were performed (Cavazza \& Poznanski, 1998) and the methods described by Di Maio et al. (2011a, 2011b) were utilised to distinguish morphologically between the Saccharomyces and Candida yeasts. Fermentations took 15 to 23 days. Sc fermentations (pure and mixed) were considered completed when the level of residual glucose was less than $1 \mathrm{~g} / \mathrm{L}$ (as assessed by Keto-Diabur-Test ${ }^{\circledR}$ 5000, Roche). Since such criterion could not be used for $\mathrm{Cz}$ pure fermentations, $\mathrm{Cz}$ strains were allowed to ferment until (and beyond) the plateau of glucose consumption.

\section{Chemical parameters, anthocyanins and colorimetric determinations}

Alcohol, reducing sugars, total acidity, colour intensity (the sum of the absorbencies at wavelengths of 420, 520 and 620 $\mathrm{nm}$ measured over a $1 \mathrm{~cm}$ path length) and hue (or tint: the ratio of the $420 \mathrm{~nm}$ and $520 \mathrm{~nm}$ absorbencies measured over a $1 \mathrm{~cm}$ path length) were determined according to EEC2676 (1990). Glucose and glucose+fructose, glycerol, acetic acid, malic acid, lactic acid, citric acid and tartaric acid were determined using an Enotech Steroglass apparatus (code SQRQ053586, Steroglass, Italy). Fructose was determined by subtracting glucose from glucose+fructose. Yeast available nitrogen (YAN) was determined according to Gump et al. (2002). Total polyphenols (TPFs), total anthocyanins and total flavonoids (in ppm) were determined according to Di Stefano et al. (1989). All measurements were performed in duplicate.

\section{RESULTS}

Characterisation of the Candida zemplinina $(\mathrm{Cz})$ isolates Several surveys have so far been performed on the yeast populations of Sicilian cultivars, none of which highlighted the presence of C. zemplinina (Castelli, 1954; Balloni \& Filpi, 1979; Favaloro et al., 1984; Pinzauti et al., 2004; Romancino et al., 2008; Capece et al., 2010 and references therein). In this work we report for the first time on the presence of Candida zemplinina strains in Sicilian grapes and musts and we provide an oenological characterisation of some representative isolates. We have conducted our analysis by considering 59 Candida isolates of the non-Saccharomyces IRVV collection. These were initially identified as 
representative of Candida stellata (Romancino et al., 2008), based on microbiological (Cavazza \& Poznanski, 1998) and molecular analyses (Esteve-Zarzoso et al., 1999; Granchi et $a l ., 1999)$. As new studies showed the prevalence of the $C$. zemplinina species in wine musts (Csoma \& Sipiczki, 2008), we reanalysed our isolates and were able to establish that all of them belonged to the $C$. zemplinina species. Sequencing of the D1/D2 domain of one of the isolates $(\mathrm{Cz} 3)$ further supported this notion (not shown).

We tested our $\mathrm{Cz}$ isolates for several oenological characteristics (see Methods). The results of these plate assays highlighted their oenological potential, showing behaviours comparable to those of the reference commercial strains. In our tests, no isolate had an $\mathrm{H}_{2} \mathrm{~S}$ production level higher than that of the references: $95 \%$ of the isolates produced mediumhigh levels; $5 \%$ produced medium levels. As for acetic acid, a greater variability was observed: $8.4 \%$ produced very low levels; $22.4 \%$ produced low levels; $6.7 \%$ produced mediumlow levels; $42.3 \%$ produced medium-high levels; $6.7 \%$ produced high levels and $13.5 \%$ produced very high levels. Some $\beta$-glucosidase activity was observed $(16.2 \%$ of the isolates had medium activity; $75 \%$ had medium-low activity; $6.7 \%$ had no activity), which was completely inhibited at sugar concentrations comparable to those of the musts (200 $\mathrm{mg} / \mathrm{L})$. No killer activity was observed, as in Comitini et al. (2011).

The mitochondrial DNA of each isolate was analysed by mt-DNA RFLP analysis and the isolates were found to belong to 14 polymorphism groups (Fig. 1). One isolate per group was then chosen to ferment $100 \mathrm{ml}$ sterile white must aliquots and assessed for the 12 oenological parameters shown in Table 1, in comparison with two commercial Saccharomyces cerevisiae (Sc) strains (L404 and NDA21). Not-inoculated must was used as a negative control ("Blank"). After two days, all the $\mathrm{Cz}$ strains had a fermentation vigour at best equal to $50 \%$ of that of the commercial Sc yeast strains: among all strains, the $\mathrm{Cz} 3$ was the one with the best $\mathrm{SO}_{2}$ tolerance value. At seven days, the fermentation vigour and the tolerance to $\mathrm{SO}_{2}$ of all the $\mathrm{Cz}$ strains increased, although they never reached the level of the references. At 30 days, almost all the $\mathrm{Cz}$ strains had fermentative power values between 8 and 11 grams of $\mathrm{CO}_{2} / 100 \mathrm{~mL}$, much lower than the Sc strains: $\mathrm{Cz} 3$ had the highest fermentative power among the Candida isolates.

Sugar consumption was lower in all the $\mathrm{Cz}$ strains compared to the Sc strains. At the end of the fermentation, the Sc strains had utilised almost all the sugars available (about $216 \mathrm{~g} / \mathrm{L}$, see "Blank"). In the $\mathrm{Cz}$ fermentations, between 50 and $100 \mathrm{~g} / \mathrm{L}$ of the sugars were left $(\mathrm{Cz} 3$ and $\mathrm{Cz} 37$ having the lowest amount), in general agreement with Magyar and Tóth (2011). Acetic acid levels of all fermentations (except $\mathrm{Cz} 1)$ were within the range defined by the two commercial Sc strains. All $\mathrm{Cz}$ yeasts were able to reduce malic acid at least to levels comparable to those of the commercial strains (most strains achieving an even greater reduction). Lactic acid levels were minimal and similar in all the fermentations analysed. Citric acid content increased sensitively at the end in all the $\mathrm{Cz}$ fermentations (a smaller increase was observed in Sc fermentations). Tartaric acid levels remained close to the blank in all fermentations.
Although the differences in glycerol between the $\mathrm{Cz}$ and the Sc fermentations were small, it should be noted that these levels were obtained with a smaller sugar consumption in the $\mathrm{Cz}$ fermentations compared to the $\mathrm{Sc}$ ones. These results would give us hope that an even higher glycerol level could be obtained in mixed $\mathrm{Cz}$-Sc fermentations, improving wines in an important positive aspect. Of all the strains, Cz3, Cz12 and Cz26 were those producing on average slightly higher amounts of glycerol. No $\mathrm{Cz}$ strain showed killer activity towards the Sc strain NDA21 in the tests performed on plates (not shown).

Based on these results, three $\mathrm{Cz}$ strains were selected for mixed fermentations trials with the Sc strain NDA21: Cz3, Cz12 and Cz26 (Table 1).

\section{Mixed fermentations in $250 \mathrm{ml}$ of must}

In what follows we present the results of single fermentation experiments conducted in $250 \mathrm{ml}$ of Nero d'Avola must (rosé). Further experiments were also performed, which confirmed the results reported here (Di Maio et al., in preparation).

In all the P fermentations, growth of the NDA21 and the $\mathrm{Cz}$ yeast strains reached a plateau before the end of the second day (close to $100 \times 10^{6} \mathrm{cfu} / \mathrm{mL}$ ) and maintained it for the next 10 or five days respectively (not shown).

In all CI fermentations, the growth of NDA21 quickly overwhelmed that of the $\mathrm{Cz}$ strains, which declined sharply. This agrees with what was reported by Comitini et al. (2011). The NDA21 growth plateau (close to $100 \times 10^{6} \mathrm{cfu} / \mathrm{mL}$ ) was reached in two to three days and was maintained until the 11th to 12th day. Even after that, the concentration of the NDA21 strain was maintained at conspicuous levels (between 5 and $10 \times 10^{6} \mathrm{cfu} / \mathrm{mL}$ ). An example of this trend is shown in Fig. 2A.

In the Seq inoculations, the growth of the $\mathrm{Cz}$ strains inoculated at day 0 reached a plateau $\left(100 \times 10^{6} \mathrm{cfu} / \mathrm{mL}\right)$ after one to two days. Considerable growth levels were observed even on the 8th or 9th day (three to four days after inoculation of the NDA21 strain). Growth of the NDA21 strain reached a plateau four to five days after its inoculation, and remained stable throughout. This is illustrated in Fig. 2B and indicates that the presence of the $\mathrm{Cz}$ yeast strains did not interfere with the growth of NDA21.

The contribution of the $\mathrm{Cz}$ strains to the variation of the chemical parameters of the must can be appreciated by looking at the results in Table 2. In the CI fermentations, the contribution of the $\mathrm{Cz}$ strains was minimal (compared to what was obtained by the inoculation of NDA21 alone: F250-1 P- NDA21). This is consistent with the result in Fig. 2A.

On the other hand, the contribution of the $\mathrm{Cz}$ strains became quite evident in the Seq fermentations. All fermentations where the NDA21 strain was present had a markedly reduced level of sugars left, while the amount of sugars left in the P-Cz fermentations (F250-2, F250-3 and F250-4) was considerable; therefore the presence of the $\mathrm{Cz}$ strains did not affect the ability of the NDA21 strain to metabolise sugars in mixed fermentations. Acetic acid increased in all the fermentations. The $\mathrm{Cz}$ strains maintained lower levels in the P fermentation; however, these increased 

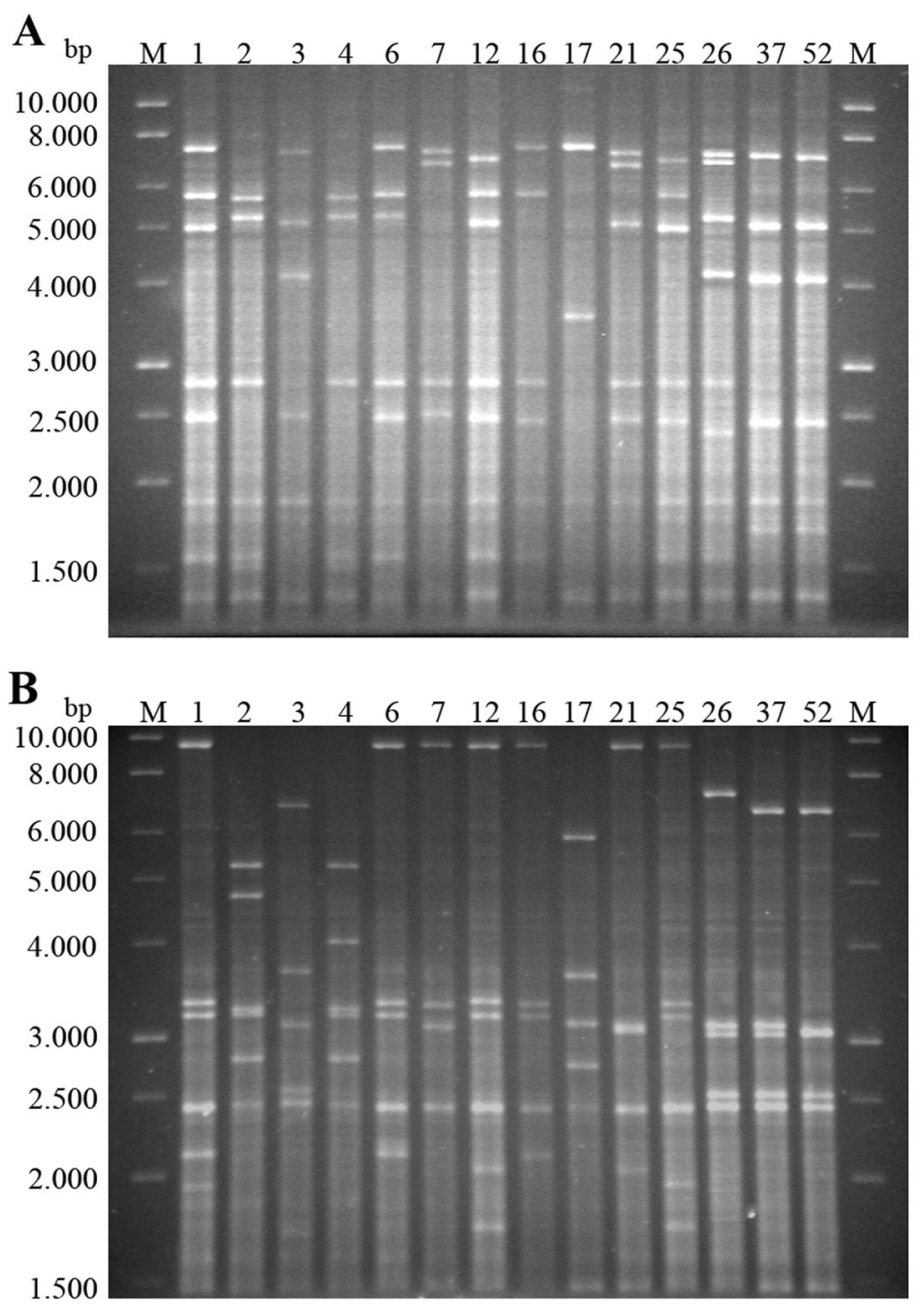

FIGURE 1

Mt-RFLP pattern analyses: one $\mathrm{Cz}$ representative isolate (indicated with its numeral) per each polymorphism group (strain) is shown. M: Molecular marker, A: HpaII pattern, B: HaeIII pattern

in all the mixed fermentations. Note that the acetic acid values found here relate to the high surface/volume ratio favouring oxidation: fermentations conducted in larger volumes produced lower acetic acid levels (Di Maio et al., in preparation).

The $\mathrm{Cz}$ strains favoured the synthesis of citric acid: this was evident in the $\mathrm{P}$ fermentations; and higher levels of citric acid were found in the Seq fermentations compared to the CI fermentations. YAN levels (in the NDA21 P and the Seq fermentations) indicated that the amount of nitrogen was enough to sustain the growth of the yeast cells. Total polyphenols (TPFs) were lower in all wines compared to the must. However, $\mathrm{Cz}$ (P and $\mathrm{Seq}$ ) wines maintained a higher TPF content than NDA21 and CI wines; the $\mathrm{Cz} 3$ wines had the highest TPF content. Anthocyanins decreased in all fermentations. The NDA21 wines had the lowest amount of these compounds, while the presence of the $\mathrm{Cz}$ strains (in the $\mathrm{P}$ or Seq fermentations) helped reduce this loss. The Cz3/NDA21 Seq fermentation showed the highest level of anthocyanins among the mixed fermentation wines.

The fructophilic character of the $\mathrm{Cz}$ isolates was assessed by monitoring the level of glucose and fructose consumption. In those fermentations in which the contribution of the Candida yeasts could be assessed (the $\mathrm{Cz}(\mathrm{P})$ and the $\mathrm{Cz}$
(Seq) fermentations before the inoculation of NDA21), the preference for fructose over glucose appeared evident: in all (P) fermentations, fructose consumption was six to 16 times higher than glucose consumption; in the (Seq) fermentations, the former was two to three times higher than the latter.

Interestingly, the contribution of the $\mathrm{Cz}$ yeasts became most evident (in the Seq-fermentations) in the observed increases in glycerol content and decreases in ethanol levels. Following our sequential inoculation protocols, glycerol levels comparable to (or even higher than) those of the $\mathrm{Cz}$ (P) fermentations were obtained. Compared to the NDA21 P fermentation, these increases were on average $3 \mathrm{~g} / \mathrm{L}$.

\section{DISCUSSION}

We have reported for the first time on the presence of $C$. zemplinina strains in Sicilian grapes and musts. Furthermore, we have presented an oenological characterisation of these strains and we have shown that at least one of them is of potential interest for the development of mixed fermentation protocols with $S$. cerevisiae strains.

As we reclassified all the Candida isolates of the Sicilian must collection (IRVV non-Saccharomyces collection) as $C$. zemplinina representatives, our results speak for the ecological prevalence of this species in Sicily and are in 

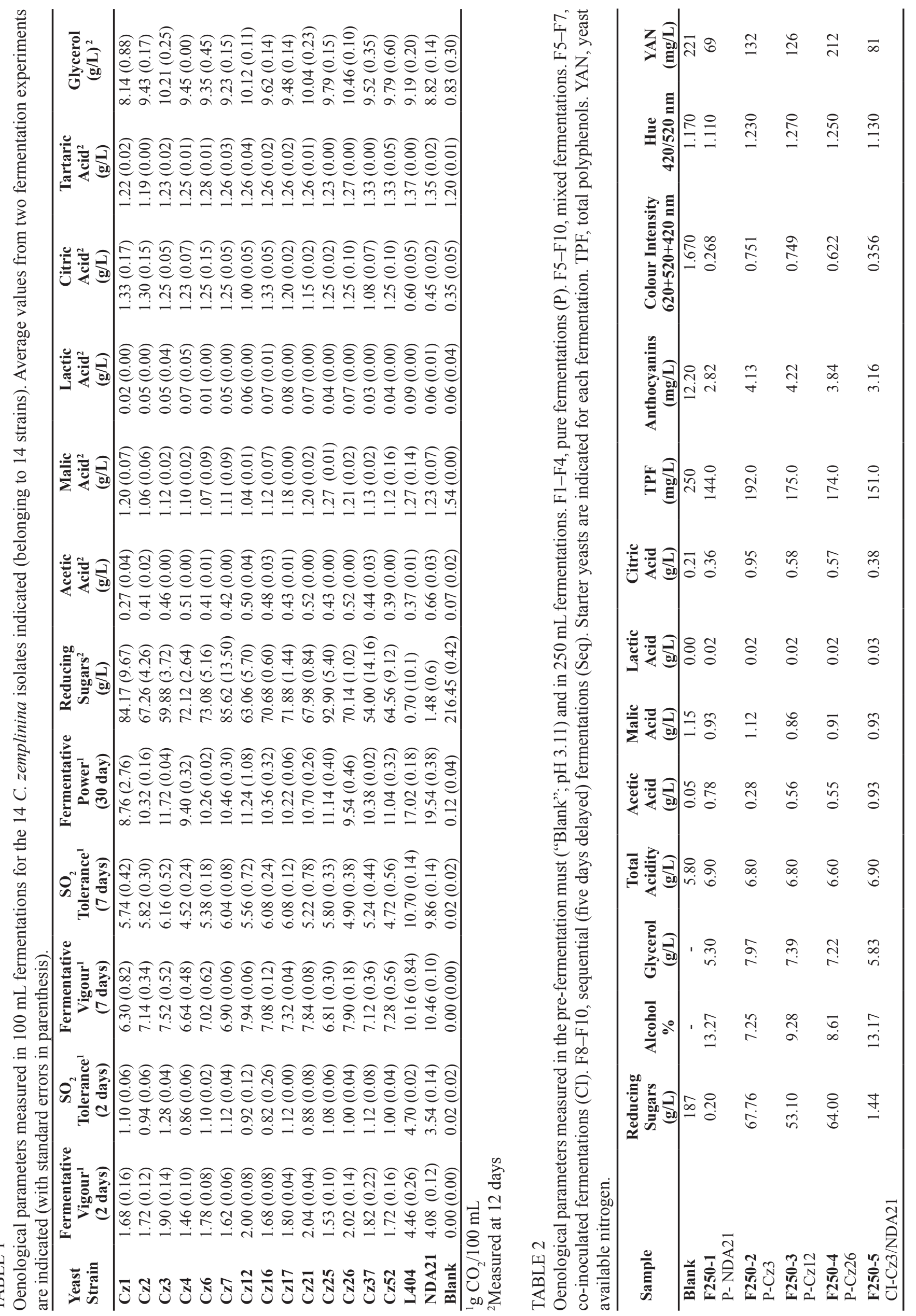

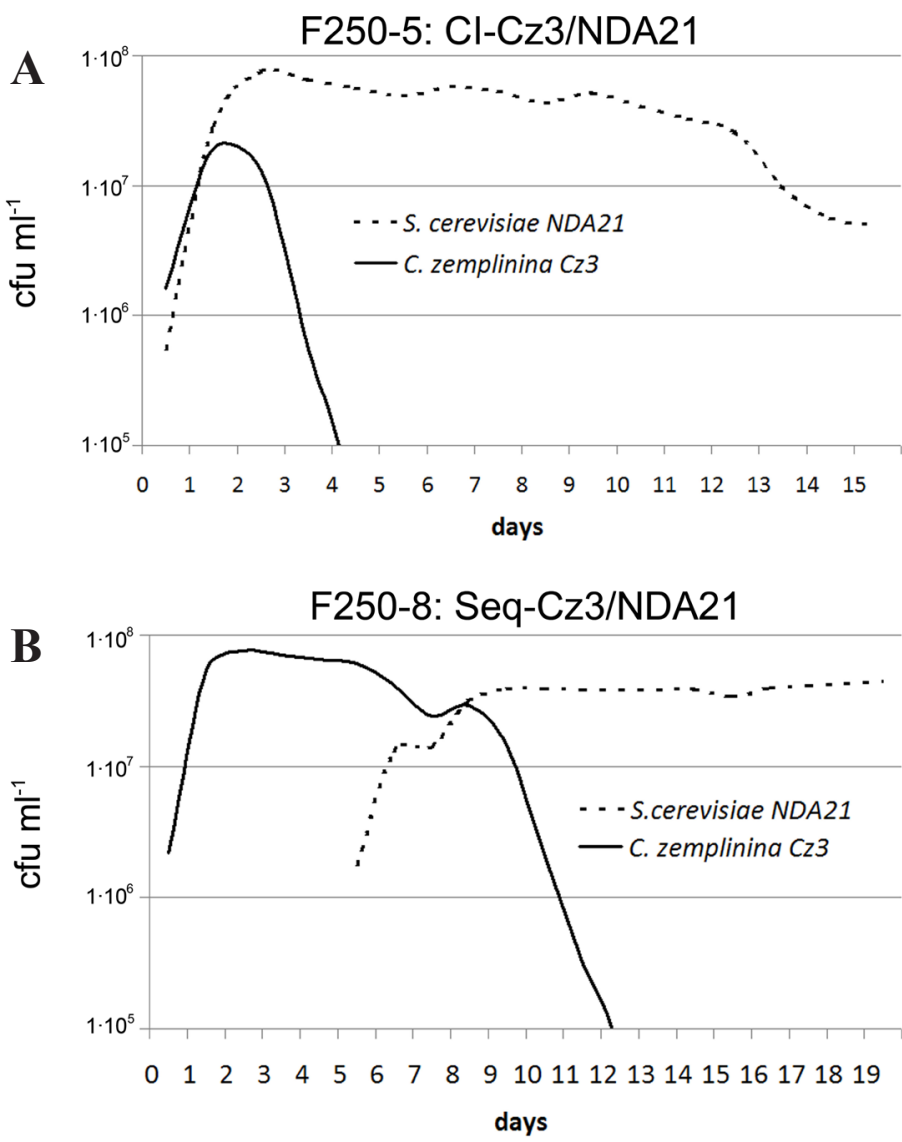

FIGURE 2

A: Growth curve for the mixed co-inoculated fermentation F250-5 (Cz3 and NDA21),

B: Growth curve for the mixed sequential fermentation F250-8 (Cz3 and NDA21).

agreement with those authors (e.g. Csoma \& Sipiczki, 2008) arguing that $C$. zemplinina (not $C$. stellata) is the most abundant Candida species on grapes and musts.

We showed that care must be taken to ensure an appropriate proliferation of both yeast starters $(\mathrm{Cz}$ and $\mathrm{Sc})$ in a mixed fermentation. In fact, in our CI experiments, the fermentation process was carried out by the Sc strain with essentially no contribution from the $\mathrm{Cz}$ strains. This is in agreement with the previous results of Comitini et al. (2011), which showed (in CI inoculations) two distinct fermentation phases (one carried out by Candida, the other by Saccharomyces) with a Cz:Sc inoculation ratio of 10 000:1. Therefore, an early, robust proliferation of Saccharomyces hinders proliferation of Candida.

On the other hand, it could be interesting to investigate how the early proliferation of Candida affects the proliferation of Saccharomyces. The levels of citric acid found at the end of all $\mathrm{Cz}$ (P and Seq) fermentations were higher than those found in the NDA21-dominated fermentations, and it has been reported that citric acid can favour Saccharomyces growth (Bardi, 2005). However, other authors have shown that, depending on $\mathrm{pH}$, citric acid might hinder Saccharomyces cerevisiae growth (Nielsen \& Arneborg, 2007). Further studies in which the parameters of the mixed fermentation are carefully taken into account 
might illuminate this issue and reveal interesting details on the yeast population dynamics occurring within the must niche.

The mixed fermentation protocol we have presented here obtains a very similar result to that already obtained by Comitini et al. (2011): an early Candida proliferation phase is followed by a later Saccharomyces one. By comparing the results of P, CI and Seq inoculations, we were able to determine the contribution made by our Candida strains. The decrease in ethanol content we obtained was comparable to that obtained by Comitini et al. (2011). However, in the Seq inoculation we had an increase in glycerol content about twice that reported by these authors. Therefore the Candida zemplinina strains we selected might be better glycerol producers, better suited for utilisation in mixed fermentation. Careful comparative studies will be needed to assess such a proposition, given the number of differences existing between these two studies (musts, starter strains utilised, inoculation protocols).

Finally, although both protocols (our Seq protocol and the 1:10000 CI inoculation of Comitini et al., 2011) obtain a similar proliferation result in sterile musts, things might work differently in a winery (where these results eventually must be applied). Several reports have shown the presence of "resident" yeasts in wineries (Ciani et al., 2004; Santamaría et al., 2005; Mercado et al., 2007); our preliminary results show that these hinder the proliferation of the yeast starters if these latter are inoculated at low concentrations (such as those proposed by Comitini et al. (2011). On the other hand, our Seq inoculation protocol allows a high concentration of both starters, possibly helping to overcome this problem. We are currently testing this proposition in the winery.

In conclusion, we have presented the oenological characterisation of the Candida zemplinina yeast strain $\mathrm{Cz} 3$, isolated in Sicily. The analysis of its technological and fermentation properties have shown that this is a promising oenological starter for mixed fermentation protocols with a potential for industrial applications.

\section{CONCLUSIONS}

We have shown the presence of Candida zemplinina yeasts in Sicilian grapes and musts. All Candida isolates of the non-Saccharomyces yeasts in the IRVV collection were representatives of the $C$. zemplinina specie. Furthermore, we have selected a $C$. zemplinina strain endowed with very promising features for future industrial applications.

\section{LITERATURE CITED}

Andorrà, I., Esteve-Zarzoso, B., Guillamón, J.M. \& Mas, A., 2010. Determination of viable wine yeast using DNA binding dyes and quantitative PCR. Int. J. Food. Microbiol. 144, 257-262.

Anfang, N., Brajkovich, M. \& Goddard, M.R., 2009. Co-fermentation with Pichia kluyveri increases varietal thiol concentrations in Savignon Blanc. Aust. J. Grape Wine Res. 15, 1-8.

Balloni, V. \& Filpi, C., 1979. Nuovo contributo alla conoscenza della zimologia dei mosti e dei vini della Sicilia occidentale. Vini d'Italia 118 , 49-58

Bardi, L., 2005. Fattori che condizionano lo sviluppo dei lieviti nella vinificazione. In: Vincenzini, M., Romano, P. \& Farris, A. (eds). Microbiologia del vino. Casa Editrice Ambrosiana, Milano, Italy.
Capece, A., Romaniello, R., Siesto, G., Pietrafesa, R., Massari, C., Poeta, C. \& Romano, P., 2010. Selection of indigenous Saccharomyces cerevisiae strains for Nero d'Avola wine and evaluation of selected starter implantation in pilot fermentation. Int. J. Food Microbiol. 144, 187-192.

Caridi, A., Cufari, J.A. \& Ramondino, D., 2002. Isolation and clonal preselection of oenological Saccharomyces. J. Gen. Appl. Microbiol. 48, 261267

Castelli, T., 1954. Les agents de la fermentation vinaire. Arch. Mikrobiol. $20,323-342$

Cavagna, M., Lorenzini, M. \& Torriani, S., 2008. Lieviti autoctoni per il Vino Santo Trentino. Terra Trentina 7, 21-26.

Cavazza, A. \& Poznanski, E., 1998. Le analisi microbiologiche nel laboratorio enologico. Vignevino, 25, 42-53.

Ciani, M., Mannazzu, I., Marinangeli, P., Clementi, F. \& Martini, A. 2004. Contribution of winery-resident Saccharomyces cerevisiae strains to spontaneous grape must fermentation. Antonie Van Leeuwenhoek, 85, 159-164.

Comitini, F., Gobbi, M., Domizio, P., Romani, C., Lencioni, L., Mannazzu, I. \& Ciani, M., 2011. Selected non-Saccharomyces wine yeasts in controlled multistarter fermentations with Saccharomyces cerevisiae. Food Microbiol. $28,873-882$

Csoma, H. \& Sipiczki, M., 2008. Taxonomic reclassification of Candida stellata strains reveals frequent occurrence of Candida zemplinina in wine fermentation. FEMS Yeast Res. 8, 328-336.

Di Maio, S., Polizzotto, G., Notarbartolo, G., Planeta, D., Genovese, S., Gurrieri, C. \& Oliva, D., 2006. Isolamento e caratterizzazione di un nuovo ceppo di lievito di interesse enologico. L'enologo, 42, 93-98.

Di Maio, S., Polizzotto, G., Planeta, D. \& Oliva, D., 2011a. A method to discriminate between the Candida stellata and Saccharomyces cerevisiae in mixed fermentation on WLD and lysine agar media. S. Afr. J. Enol. Vitic. $32,35-41$

Di Maio, S., Polizzotto, G., Planeta, D. \& Oliva, D., 2011b. Errata corrige on: A method to discriminate between the Candida stellata and Saccharomyces cerevisiae in mixed fermentation on WLD and lysine agar media. S. Afr. J. Enol. Vitic. In press.

Di Maio, S., Polizzotto, G., Di Gangi, E., Foresta, G., Genna, G., Verzera, A., Scacco, A., Amore, G. \& Oliva, D., 2012. Biodiversity of indigenous Saccharomyces populations from old wineries of South-Eastern Sicily (Italy): Preservation and economic potential. PLoS ONE 7(2): e30428, doi:10.1371/journal.pone.0030428.

Di Stefano, R., Cravero, M.C. \& Gentilini, N., 1989. Metodo per lo studio dei polifenoli dei vini. L'enotecnico, 5, 83, 89.

Du Toit, M. \& Pretorius, I.S., 2000. Microbial spoilage and preservation of wine: using weapons from nature's own arsenal - a review. S. Afr. J. Enol. Vitic. 21, 74-96

EEC 2676, 1990. Commission regulation determining community methods for the analysis of the wines

Esteve-Zarzoso, B., Belloch, C., Uruburu, F. \& Querol, A., 1999. Identification of yeasts by RFLP analysis of the 5.8S rRNA gene and the two ribosomal internal transcribed spacers. Int. J. Syst. Bact. 49, 329-337.

Favaloro, M., Scalici, G. \& Balloni, W., 1984. Evoluzione della blastofora nella fermentazione dei mosti a bassa temperatura in Sicilia. Atti dell'Accademia Italiana della vite e del vino. Vol. XXXVI.

Granchi, L., Bosco, M., Messini, A. \& Vincenzini, M., 1999. Rapid detection and quantification of yeast species during spontaneous wine fermentation by PCR-RFLP analysis of the rDNA ITS region. J. Appl. Microbiol. 87, 949-956.

Gump, B.H., Zoecklein, B.W., Fugelsang, K.C. \& Whiton, R.S., 2002. Comparison of analytical methods for prediction of prefermentation nutritional status of grape juice. Am. J. Enol. Vitic. 53, 325-329. 
Languet, P., Aguera, E., Samson, A., Ortiz-Julien, A. \& Salmon, J.M., 2005. Valorization aromatique des mouts par l'utilisation séquentielle des levures d'espécies non-Saccharomyces et Saccharomyces. Rev. Oenolg. 117, 31-33.

Magyar, I. \& Tóth, T., 2011. Comparative evaluation of some oenological properties in wine strains of Candida stellata, Candida zemplinina Saccharomyces uvarum and Saccharomyces cerevisiae. Food Microbiol 28, 94-100.

Mercado, L., Dalcero, A., Masuelli, R. \& Combina, M., 2007. Diversity of Saccharomyces strains on grapes and winery surfaces: analysis of their contribution to fermentative flora of Malbec wine from Mendoza (Argentina) during two consecutive years. Food Microbiol. 24, 403-412.

Nickerson, W.J., 1953. Reduction of inorganic substances by yeasts. I Extracellular reduction of sulfite by species of Candida. J. Infect. Dis. 93 43-56.

Nielsen, M.K. \& Arneborg, N., 2007. The effect of citric acid and pH on growth and metabolism of anaerobic Saccharomyces cerevisiae and Zygosaccharomyces bailii cultures. Food Microbiol. 24, 101-105.

Pinzauti, F., Sebastiani, F., Giannone, V., Lombardo, A. \& Barberio, C., 2004. Selection and characterization of wine yeast strains isolated from different areas in Sicily. Rivista di Viticoltura ed Enologia 57, 69-81.

Pramateftaki, P.V., Lamaridis, P. \& Typas, M.A., 2000. Molecular identification of wine yeast at species or strain level: a case study with strains from two vine-growing areas of Greece. J. Appl. Microbiol. 89, 236248.
Querol, A., Barrio, E., Huerta, T. \& Ramòn, D., 1992. Molecular monitoring of wine fermentations conducted by active dry yeast strains. Appl. Environ. Microbiol. 58, 2948-2953.

Rantsiou, K., Boundy-Mills, K. \& Cocolin, L., 2008. Lieviti fruttosofili ad interesse enologico. Quad. Vitic. Enol. Univ. Torino 30, 99-109.

Regodón, J.A., Peréz, F., Valdés, M.E., De Miguel, C. \& Ramírez, M., 1997. A simple and effective procedure for selection of wine yeast strains. Food Microbiol. 14, 247-254.

Romancino, D.P., Di Maio, S., Muriella, R. \& Oliva, D., 2008. Analysis of non-Saccharomyces yeast populations isolated from grape musts from Sicily (Italy). J. Appl. Microbiol., 105, 2248-54

Santamaría, P., Garijo, P., López, R., Tenorio, C. \& Rosa Gutiérrez, A., 2005. Analysis of yeast population during spontaneous alcoholic fermentation: effect of the age of the cellar and the practice of inoculation. Int. J. Food Microbiol. 103, 49-56.

Strauss, M.L., Jolly, N.P., Lambrechts, M.G. \& Van Rensburg, P., 2001. Screening for the production of extracellular hydrolytic enzymes by nonSaccharomyces wine yeasts. J Appl Microbiol 91, 182-190.

Zambonelli, C., Tini, V. \& Castellari, L., 2000. Guida all'uso dei lieviti selezionati in enologia, Calderini Edagricole, Bologna, Italy. 